\title{
Influencia de la ayuda del profesorado en la construcción del modelo de sistema inmunológico y su aplicación en las tomas de decisión
}

\author{
Araitz Uskola $^{1, \mathrm{a}}$, Begoña Burgoa ${ }^{2, \mathrm{~b}}$, Gurutze Maguregi ${ }^{1, \mathrm{c}}$ \\ ${ }^{1}$ Departamento de Didáctica de la Matemática y de las Ciencias Experimentales, Facultad de Educación de \\ Bilbao, Universidad del País Vasco UPV/EHU. 48940 Leioa \\ ${ }^{2}$ Instituto de Evaluación e Investigación Educativa ISEI-IVEI (Gobierno Vasco), LIEC (Universitat Autònoma de \\ Barcelona) \\ aaraitr.uskola@ebu.eus,bbbetxaburu@gmail.com,_gurutre.maguregi@ebu.eus
}

[Recibido: 19 Junio 2017. Revisado: 20 Diciembre 2017. Aceptado: 4 Junio 2018]

\begin{abstract}
Resumen: En este trabajo se analiza la construcción del modelo de sistema inmunológico por parte de estudiantes y su transferencia a otros contextos, mediante diversas situaciones contextualizadas, que parten de una controversia sociocientífica sobre la vacunación. Se estudia cómo influye la ayuda del profesorado en la construcción de dicho modelo y el desarrollo de la competencia argumentativa en la toma de decisiones sobre aspectos de salud relacionados con el citado modelo. El estudio compara datos de las producciones escritas y las discusiones recogidas durante dos años en el Grado de Educación Primaria. Los resultados muestran mayor nivel del modelo construido y transferido el segundo año, que podría deberse a los cambios en las actividades y en la acción de las profesoras. La competencia argumentativa mejora en los dos cursos, hallando diferencias según sea la toma de decisión de ámbito personal o social.
\end{abstract}

Palabras clave: Modelo; Rol del profesorado; Sistema inmunológico

Influence of the teacher's guide in the construction of the immune system model and its application in decision making

Abstract: This work analyzes the construction of the immune system model by students beginning with several contextualized situations stated after reading a socioscientific controversy about vaccination, and its transference to other contexts. It is studied how the teacher's guiding has influence in the construction of the model. Besides, the argumentative competence while making decisions related to the model is investigated. The written productions and the oral discussions among elementary pre-service teachers during two different years were compared. The results show higher level in the model constructed and transferred the second year, which could be attributed to the changes in activities and in actions taken by the teachers. The argumentative competence improves both years, differences depending on the decision makings being personal or social are found and discussed.

Keywords: Model; Role of the teacher; Immune system

Para citar este artículo: Uskola, A., Burgoa, B., Maguregi, G. (2018) Influencia de la ayuda del profesorado en la construcción del modelo de sistema inmunológico y su aplicación en las tomas de decisión. Revista Eureka sobre Enseñanza y Divulgación de las Ciencias 15 (3), 3604.doi:10.25267/Rev_Eureka_ensen_divulg_cienc.2018.v15.i3.3604

\section{Introducción}

La ciencia impregna casi todas las facetas de la vida moderna y tiene la clave para alcanzar muchos de los retos más urgentes de la humanidad. Sin embargo, mucha población joven carece de un conocimiento sólido de ésta (NRC 2012). Los países que participan en la evaluación PISA comparten el pensamiento de que la comprensión de la ciencia y la competencia científica son tan importantes que deberían ser aspectos de la educación para todas las personas. Se define la competencia científica como "la habilidad de comprometerse con cuestiones relacionadas con la ciencia y con las ideas científicas, como un ciudadano reflexivo” (OCDE 2013, p.7). La persona competente científicamente tiene que ser capaz de

\author{
Revista Eureka sobre Enseñanza y Divulgación de las Ciencias \\ Universidad de Cádir. APAC-Eureka. ISSN: 1697-011X \\ bttp:/ / dx.doi.org/10.25267/Rev_Eureka_ensen_divulg_cienc.2018.v15.i3.3604 \\ bttp:/ / reuredc.uca.es
}


explicar fenómenos científicamente, evaluar y diseñar investigaciones científicas, e interpretar científicamente datos y evidencias.

Tanto la investigación en didáctica de las ciencias como los nuevos marcos curriculares señalan la participación del alumnado en las prácticas científicas como vía para desarrollar la competencia científica. Así, para reconocer y explicar los fenómenos naturales es necesario identificar, utilizar y generar modelos explicativos y representaciones asociadas a los mismos, modelizar (Schwarz et al. 2009), y para interpretar científicamente datos, es necesario implicarse en actividades de argumentación, tales como tomas de decisiones basadas en datos (Jiménez-Aleixandre 2010).

El presente estudio parte de los resultados y conclusiones de una investigación llevada a cabo en 2015/16 (Maguregi, Uskola y Burgoa 2017). En ella se observaron dificultades en la transferencia del modelo de sistema inmunológico por parte del alumnado del Grado de Educación Primaria y se propusieron cambios en la ayuda del profesorado, planteando diversidad de contextos y promoviendo la generalización y abstracción del modelo.

\section{Marco teórico}

\section{Modelos y modelización}

Una persona científicamente competente se basa en modelos científicos y sus representaciones para tratar de explicar y predecir fenómenos científicos (Schwarz et al. 2009). Para Gilbert (2004) el modelo es una representación estructurada que incluye elementos simbólicos de las características principales de una idea, objeto, proceso o sistema. Además, la explicitación de los modelos por parte del estudiante permite a los docentes acceder a las ideas del alumnado y conocer cómo evolucionan (Mendonça y Justi 2014), ya que los modelos mentales son dinámicos y evolucionan permanentemente al interactuar con el contexto (Alzate 2013). Por ello, para Gilbert (2004) los modelos son, a la vez que productos de la ciencia, las principales herramientas del aprendizaje y la enseñanza.

El proceso de construcción de modelos mentales o modelización se genera mediante los razonamientos y experiencias (Mendonça y Justi 2014). Las actividades de modelización que se realizan en la clase de ciencias han de incluir: explorar, expresar, construir, aplicar y revisar modelos (Marchán-Carvajal y Sanmartí 2015).

El concepto de transferencia ha sido acunado para designar la utilización del conocimiento adquirido en un contexto específico a una nueva situación. Desde la perspectiva Actor Oriented Transfer-AOT (Lobato 2012), se conceptualiza la transferencia no solo como la aplicación del modelo mental que una persona lleve a cabo (aplicación estática del conocimiento), sino como la reconstrucción dinámica del conocimiento aprendido que la persona realiza en la nueva situación. Barnett y Ceci (2002) distinguen dos tipos de transferencia, la cercana y la lejana. Se considera transferencia cercana cuando la nueva situación se encuentra próxima o es similar a la de aprendizaje, y transferencia lejana en caso contrario.

\section{El modelo de sistema inmunológico en relación con el modelo de ser vivo}

El modelo de ser vivo es considerado tanto desde las ciencias como desde la ciencia escolar como uno de los grandes modelos a construir. Sobre el modelo de ser vivo en la ciencia escolar (Pigrau y Sanmartí 2015), los autores crearon uno referido al sistema inmunológico que se muestra en la figura 1 (Maguregi et al. 2017). 


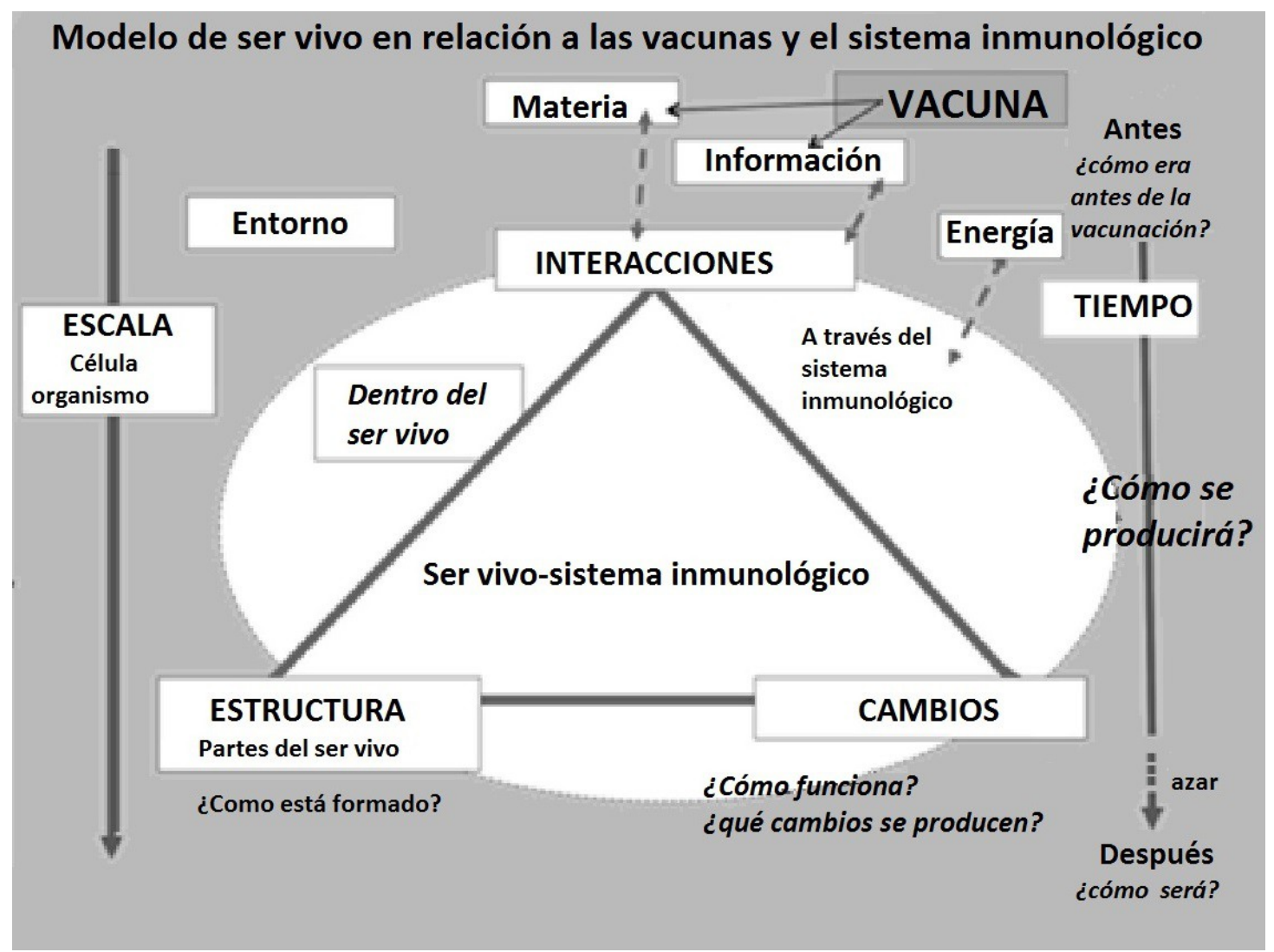

Figura 1. Modelo de Sistema Inmunológico (SI) en relación con el modelo de ser vivo (Maguregi et al. 2017). Se representa el ser vivo como formado por una estructura diferenciada del entorno y en interacción con él. El SI está formado por parte de la estructura del ser vivo y toma parte en la interacción, cuando los elementos externos se internan en la estructura del ser vivo. Esto produce cambios en el propio SI.

Andrade, Araújo-Jorge y Coutinho-Silva (2016) analizaron las dificultades de los estudiantes para comprender el funcionamiento del sistema inmunológico y hallaron que la mayoría de los estudiantes atribuían a este acciones de defensa destinadas a la protección del organismo ante la invasión de patógenos. Verificaron un limitado conocimiento respecto a las interacciones del organismo consigo mismo y con el entorno para la consecución del equilibrio. El modelo de sistema inmunológico propuesto pretende avanzar desde las concepciones más beligerantes, mayoritarias desde 1960, hacia un modelo que se centre en su papel en el mantenimiento de la estructura o equilibrio del cuerpo (Andrade et al. 2016).

Entre los trabajos que investigan el modelo del sistema inmunológico que construyen los futuros docentes cabe destacar los realizados por Aznar y Puig (2016a, 2016b) sobre la tuberculosis, con dos grupos de estudiantes, uno de ellos afectado por la enfermedad. Las investigadoras constataron que el grupo afectado presentaba una mayor comprensión sobre la enfermedad, y que ambos grupos, al final, consideraban las vacunas como agentes preventivos y no curativos. Aún así, concluyeron que las ideas del alumnado no llegaban al modelo científico de referencia.

\section{Argumentación}

Argumentar, es decir, relacionar las afirmaciones realizadas con las pruebas disponibles, evaluar las teorías en función de dichas pruebas constituye parte esencial de la competencia científica (OCDE 2013).

Hay varias formas de evaluar la calidad de la argumentación, dependiendo del contexto en que se produce. Cuando se trata de cuestiones sociocientíficas en las que se toma una decisión 
justificada, uno de los aspectos clave es la capacidad de construir un contraargumento, lo que implica construir refutaciones (Erduran, Simon y Osborne 2004). Otro de los criterios que determinan la calidad argumentativa es la capacidad de pensar en otra perspectiva, en las limitaciones de la propia opción o las ventajas de las otras (Kortland 1996). Es por eso interesante que, al igual que en las situaciones reales, las opciones presenten ventajas y desventajas, de tal forma que en la decisión final, además de tenerlas en cuenta, el alumnado establezca una jerarquía de valores (Jiménez-Aleixandre 2010). Así lo hicieron García-Milá, Gilabert, Erduran y Felton (2013), y observaron en su estudio que los argumentos posteriores a la discusión en parejas eran de mayor calidad que los escritos anteriormente y mejoraban especialmente a la hora de justificar la opción escogida y de reconocer las limitaciones de la propia opción, aspecto que incluyeron en las refutaciones.

\section{El rol del profesorado como guía del aprendizaje}

Son varios los autores que señalan la necesidad de guiar a los estudiantes para que se produzca aprendizaje conceptual en ciencias. Kirschner, Sweller y Clark (2006) criticaron las propuestas constructivistas poco guiadas, basándose en cómo funcionan la memoria de trabajo, la memoria a largo plazo, y en investigaciones previas. Concluyeron que sin guía los estudiantes pueden adquirir un conocimiento desorganizado o incompleto o incluso errores conceptuales. Hardy, Jonen, Möller y Stern (2006) indicaron que en actividades muy abiertas, los estudiantes pueden centrarse en la actividad en sí y no reflexionar acerca de los conceptos relevantes, por lo que pueden no llegar a las conclusiones científicas que se pretendían porque no le dan sentido dentro de un marco conceptual. Tras hacer una revisión de las ayudas instruccionales consideradas como andamiaje, identificaron dos elementos clave: 1) estructurar las actividades para mantener a los estudiantes centrados en aspectos relevantes; 2) facilitar la reflexión de los estudiantes dentro de un contexto de razonamiento científico.

\section{Centrar a través de actividades}

Criswell (2012) estableció un marco para situar el andamiaje del profesor, uno de cuyos elementos es el contexto, que representa el gancho que capta el interés del estudiante y le da razones para invertir en el aprendizaje. La importancia del uso de contextos en el aprendizaje de las ciencias ha sido investigada (Izquierdo, Caamaño y Quintanilla 2007; Marchán-Carvajal y Sanmartí 2014) y se concluye que el contexto debe crear conexiones con el curriculum, lo que hará que los estudiantes tiendan más fácilmente a utilizar el conocimiento generado en actividades previas como recurso para la presente (Criswell 2012). Así, se puede presentar un concepto en varios contextos o áreas para favorecer su interrelación y establecer similitudes (Bransford y Schwartz 1999), de manera que se disponga de un cuerpo de conocimiento rico y contextualizado (Alexander y Murphy 1999) y se incremente la posibilidad de trasferencia (Carpintero 2002).

\section{Facilitar la reflexión: Ayudar a abstraer y generalizar}

Schalk, van der Schee y Boersma (2013) observaron que los estudiantes en la mayoría de los casos sólo llegaban a realizar generalizaciones y explicaciones con la ayuda del profesor y, sin ella, se quedaban en el nivel de las descripciones. También Zangori, Forbes y Schwarz (2015) estudiando el diferente nivel conceptual sobre el ciclo del agua alcanzado por estudiantes de Primaria que habían recibido andamiaje y que no, hallaron que solo llegaban al nivel más alto los que sí habían recibido. En el estudio de Hardy et al. (2006) se comparaba la comprensión conceptual acerca de la densidad y la flotación en estudiantes de Primaria, unos con una alta ayuda instruccional y otros con baja. La ayuda incluía actividades acotadas e intervención del profesorado para facilitar la generalización. Hallaron que, a pesar de que todos mostraban mejora en la comprensión, ésta era más estable a largo plazo en los que habían recibido una 
alta ayuda, lo que indicaba una comprensión más coherente. Decristan et al. (2015), en un amplio estudio en Primaria, hallaron que los estudiantes que recibían un tratamiento adicional en una unidad sobre la flotación y la densidad, alcanzaban una mejor comprensión de estos conceptos.

\section{Objetivos}

Este estudio pretende sumarse a los que han analizado la influencia de la ayuda ofrecida por el profesorado en la construcción de modelos por parte del alumnado. Las preguntas de la investigación son:

- ¿Qué modelo de sistema inmunológico construyen en el contexto de la vacunación y cómo lo transfieren a otros contextos? ¿Cómo influye la guía prestada por las profesoras en el modelo construido y su transferencia?

- ¿Qué competencia argumentativa manifiestan en la toma de decisiones sobre controversias de tipo personal y social relacionadas con la salud en la que está implicado el funcionamiento del sistema inmunológico?

\section{Metodología}

La investigación se ha realizado en dos cursos. En 2015/16, el equipo formado por los autores diseñó una secuencia de actividades sobre la vacunación (Maguregi et al. 2017), que fue implementada por una de ellas en una clase de $4^{\circ}$ curso del Grado de Educación Primaria. En 2016/17, se modificó la propuesta y fue implementada por dos profesoras del equipo (esto no fue posible en 2015/16), cada una en una clase distinta de $4^{\circ}$ curso del Grado de Educación Primaria.

Para responder a las preguntas de la investigación, se ha seguido una metodología de carácter interpretativo (Erickson 1989). Para responder a la primera pregunta se han categorizado las respuestas y las tres investigadoras han realizado el análisis de la muestra completa de manera independiente, para validar el análisis (Gee 1999). Las discrepancias no han superado el 15\% y se ha llegado a un consenso a través de la discusión de las interpretaciones.

En cuanto a la segunda pregunta, se analizó una cuarta parte de la muestra de forma conjunta entre las tres autoras, poniendo en común todos los resultados, y llegando a acuerdos en los casos de falta de consenso. El resto de la muestra se ha analizado de forma independiente entre los tres autores, consensuando las interpretaciones complicadas.

En los casos en que se ha determinado si hay diferencias estadísticamente significativas entre las situaciones inicial y final, se han calculado los tamaños de efecto utilizando la d de Cohen. Para ello, en primer lugar se ha determinado si se podía eliminar la hipótesis nula (no hay efecto) utilizando el test no paramétrico de Wilcoxon para variables de escala, para lo que se ha utilizado el software SPSS. A continuación se ha calculado la d de Cohen como la diferencia de los promedios dividido entre la desviación estándard en el cuestionario final. Hay que tener en cuenta que suele considerarse que un valor de d mayor a 0,5 está asociado a un tamaño de efecto moderado, y uno de 0,8 o mayor, a un efecto grande (Morales 2011).

\section{Participantes}

Los participantes en 2015/16 fueron 42 estudiantes (21,7 años de media, 85,7\% mujeres), de $4^{\circ}$ curso del Grado de Educación Primaria, que cursaban la asignatura "Nuevas Tendencias en la Didáctica de las ciencias". La secuencia se implementó en Octubre-Diciembre de 2015. Se 
dedicaron un total de 6,5 horas de clase a las actividades. Para las actividades grupales se formaron 9 grupos con 4-5 miembros.

En 2016/17 los participantes fueron un total de 79 estudiantes (22,3 años de media, 62\% mujeres) de dos clases distintas. La secuencia se implementó entre Octubre-Diciembre de 2016. Se dedicaron un total de 9 horas de clase a las actividades. El aumento respecto al 2015/16 se debió a las horas dedicadas en clase a la construcción y presentación de las maquetas. En las actividades grupales, se dividieron en 18 grupos, de 4-5 miembros.

Los nombres de los estudiantes se han sustituido por pseudónimos con la forma nAn o nOn, en los que el primer número indica el curso (1: 2015/16, 2: 2016/17): A, si son mujeres; u O, en el caso de que sean hombres; y, por último el número del estudiante ordenado alfabéticamente.

\section{Secuencia de actividades}

La secuencia de actividades en los dos cursos se detalla en la Tabla 1. En la Fase 1, las actividades fueron similares en los dos cursos, pero una vez compartida la información en la Fase 2, en 2016/17 se realizaron modificaciones teniendo en cuenta los pobres resultados en la transferencia obtenidos en 2015/16 (Maguregi et al. 2017). Siguiendo a varios autores (Decristan et al. 2015, Hardy et al. 2006), la ayuda de las profesoras tuvo como objetivos:

- Centrar la actividad cognitiva del alumnado a través del diseño de actividades. Así, las actividades introducidas fueron primeramente pensar en diversas situaciones en las que actuara el sistema inmunológico, para a continuación, buscar información, construir y explicar representaciones (maquetas) de dichas situaciones. Las situaciones fueron enfermedades infecciosas, herida infectada, vacunación y alergias. Los estudiantes diseñaron y construyeron con materiales que adquirieron de forma autónoma, una maqueta que representaba los elementos y procesos a nivel macro y micro.

- Ayudar al alumnado a abstraer y generalizar el funcionamiento del sistema inmunológico. Esto se ha llevado a cabo abriendo espacios para que el alumnado, después de la búsqueda de información sobre la vacunación, planteara situaciones que podían servir de contextos para construir un modelo de sistema inmunológico contextualizado, preguntando y señalando aspectos clave mientras los grupos realizaban sus representaciones, y ayudando a buscar semejanzas entre los procesos, tras la representación y explicación de dichas situaciones.

En la Fase 3: Transferencia, en ambos cursos, se plantearon actividades en las que se buscaba la aplicación del conocimiento construido en situaciones cercanas a los contextos trabajadostransferencia cercana -, así como la transferencia lejana en una situación de tatuaje. Así mismo, durante esta última fase se les solicitaba tomar decisiones justificadas relacionadas con la vacunación. Estas últimas actividades estaban diseñadas para favorecer la argumentación, ya que las tomas de decisión, en especial las grupales, suponen una actividad adecuada para justificar las opiniones propias (Jiménez-Aleixandre 2010). 
Tabla 1. Secuencias de actividades en torno a la vacunación implementadas en 2015/16 y 2016/17.

\begin{tabular}{c|c|c|c}
\hline \multirow{2}{*}{ Fase 1: } & \multicolumn{2}{|c}{ Fase 2: } & Fase 3: \\
& \multicolumn{2}{|c}{ Reestructuración } & Transferencia \\
\hline $2015 / 16$ y 2016/17 & $2015 / 16$ & $2016 / 17$ & $2015 / 16$ y 2016/17 \\
\hline $\begin{array}{c}\text { Explicitar conocimiento/ideas } \\
\text { sobre el SI y su funcionamiento }\end{array}$ & \multicolumn{2}{|c|}{$\begin{array}{c}\text { Búsqueda e intercambio de } \\
\text { información }\end{array}$} & $\begin{array}{c}\text { Aplicación del modelo y } \\
\text { transferencia cercana y lejana }\end{array}$ \\
\hline Toma de Decisión Personal & \multicolumn{2}{|c|}{$\begin{array}{c}\text { Construcción de } \\
\text { maquetas sobre una } \\
\text { situación } \\
\text { relacionada con el } \\
\text { funcionamiento del } \\
\text { SI }\end{array}$} & Toma de Decisión Personal \\
\hline Formular preguntas & & $\begin{array}{c}\text { Generalización del } \\
\text { modelo }\end{array}$ & Toma de Decisión Social \\
\hline
\end{tabular}

\section{Instrumentos y métodos de análisis}

\section{Modelo de sistema inmunológico construido}

Para conocer qué modelo de sistema inmunológico construye el alumnado se han analizado las respuestas individuales escritas a las preguntas ¿Por qué no se pasa la varicela más de una vez?, Explica qué pasa en tu cuerpo cuando se infecta una herida y ¿Qué ocurre en tu cuerpo cuando te vacunas?, de la Fase 1 (denominadas c1varicela, c1herida y c1vacuna), las discusiones de los estudiantes mientras intercambiaban información (denominada inf en la figura 2) y en la presentación de las maquetas (denominada maqueta en la figura 3), de la Fase 2, y las respuestas individuales a las preguntas Explica el funcionamiento de las vacunas, Explica cómo trabaja el sistema inmunológico y ¿Qué ocurre en tu cuerpo cuando te tatúas? (c3vacuna, c3SI y c3tatu), de la Fase 3.

Las categorías establecidas están relacionadas con la integración de los distintos componentes del modelo de ser vivo, en relación al sistema inmunológico (Tabla 2). Así, el nivel SI0 engloba las respuestas incorrectas que consideran al cuerpo como inerte; SI1 refleja alusiones a los componentes, a la estructura del sistema, además de los cambios morfológicos que sufre el cuerpo como inflamación, aumento de temperatura y presencia de plaquetas; SI2 incluye menciones a los cambios, a que el cuerpo reacciona produciendo anticuerpos; SI3 recoge alusiones al cuerpo como un sistema que actúa a lo largo del tiempo una vez producidos los anticuerpos.

Se han leído las producciones anteriormente descritas, analizando todas las frases y categorizando cada producción según el mayor nivel mostrado. En el caso de las producciones grupales (discusiones y presentaciones) se ha categorizado al grupo de la misma manera. 
Tabla 2. Categorías de expresiones del modelo de Sistema Inmunológico (SI) en relación al modelo de ser vivo

\begin{tabular}{|c|c|c|c|}
\hline Nivel & Descripción & $\begin{array}{l}\text { Ejemplos SI EN CONTEXTO } \\
\text { DE LA VACUNACIÓN }\end{array}$ & Ejemplos SI EN OTROS CONTEXTOS \\
\hline SI3 & $\begin{array}{l}\text { El cuerpo } \\
\text { produce } \\
\text { anticuerpos y su } \\
\text { función a lo } \\
\text { largo del } \\
\text { tiempo }\end{array}$ & $\begin{array}{l}\text { 1O19: Una vacuna tiene virus } \\
\text { atenuados. Cuandor se } \\
\text { introducen en el cuerpo, } \\
\text { nuestro cuerpo prepara un } \\
\text { ataque inmunológico para } \\
\text { destruir el virus, produciendo } \\
\text { anticuerpos específicos. En ese } \\
\text { momento nuestro cuerpo } \\
\text { reconocerá ese nuevo virus y } \\
\text { sabrá "cómo eliminarlo" }\end{array}$ & $\begin{array}{l}\text { 2A31: Cuando te haces un tatuaje en el brazo, para } \\
\text { hacerlo la aguja te hace una herida para que la tinta } \\
\text { entre debajo de la piel. (...) En ese momento el } \\
\text { sistema inmunológico de nuestro cuerpo se pone en } \\
\text { marcha (...). El cuerpo hace frente a la sustancia } \\
\text { desconocida inyectada (tinta), envía glóbulos } \\
\text { blancos a la zona de la herida, para hacer frente a las } \\
\text { bacterias que han entrado y evitar la infección. (...) } \\
\text { En todo este proceso, el cuerpo produce } \\
\text { anticuerpos para hacer frente en un futuro a este } \\
\text { tipo de bacterias. }\end{array}$ \\
\hline SI2 & $\begin{array}{l}\text { El cuerpo } \\
\text { produce } \\
\text { defensas o } \\
\text { anticuerpos }\end{array}$ & $\begin{array}{|lrr|}\text { 2A1: } & \text { Nuestro cuerpo recibe } \\
\text { virus } & \text { inactivos, } & \text { y } \\
\text { consecuencia } & \text { produce } \\
\text { anticuerpos para hacerles frente. }\end{array}$ & $\begin{array}{l}\text { OO37: Para hacer un tatuaje tenemos que agujerear } \\
\text { nuestra piel con una aguja. (...)En esa herida toman } \\
\text { parte los glóbulos blancos. Junto con la aguja } \\
\text { también entra la tinta en la herida, que se queda en } \\
\text { a piel. Nuestro SI se pone en marcha, es decir, los } \\
\text { glóbulos blancos irán a la zona de la herida. (...) }\end{array}$ \\
\hline SI1 & $\begin{array}{l}\text { El cuerpo posee } \\
\text { defensas, el } \\
\text { cuerpo sufre } \\
\text { cambios } \\
\text { morfológicos }\end{array}$ & $\begin{array}{l}\text { 2A57: Los anticuerpos que } \\
\text { tenemos en el cuerpo hacen } \\
\text { frente a los virus. }\end{array}$ & $\begin{array}{l}\text { 2O53: Cuando se infecta una herida en nuestro } \\
\text { cuerpo, se inflama la zona infectada y las "defensas" } \\
\text { de nuestro cuerpo no son capaces de curarla. }\end{array}$ \\
\hline SI0 & $\begin{array}{l}\text { Respuestas } \\
\text { incorrectas: } \\
\text { El cuerpo es } \\
\text { inerte }\end{array}$ & $\begin{array}{l}\text { 1A11: Al tomar vacunas } \\
\text { introducimos en nuestro cuerpo } \\
\text { anticuerpos y conseguimos } \\
\text { defensas contra ese virus. }\end{array}$ & $\begin{array}{l}\text { OO79: Cuando se infecta una herida entran } \\
\text { microorganismos y bacterias en nuestro cuerpo, } \\
\text { dificultando la curación de la herida. }\end{array}$ \\
\hline
\end{tabular}

\section{Competencia argumentativa}

En cuanto al estudio de la capacidad argumentativa se ha analizado, por una parte, la calidad de la argumentación, tomando como base la presencia de distintos elementos (Toulmin 1958) en los argumentos (justificaciones, datos, limitaciones); y, por otra, el tipo de justificaciones empleados en las tomas de decisiones (en adelante, TD). Se han analizados los argumentos generados en las TD de tipo personal y de tipo social.

La TD Personal de la Fase 3 consistió en decidir si pondrían o no la vacuna a su hija una vez conocidos los problemas asociados al virus del papiloma humano. Para el estudio, se ha realizando el contraste con la Fase 1, en que se les preguntaba si estaban a favor o en contra de la vacunación.

La TD Social de la Fase 1 consistió en manifestar la postura frente a la obligatoriedad de las vacunas. En la Fase 3, en 2015/16, consistió en una discusión grupal en la que tenían que decidir como profesorado de un claustro si llevarían a una salida a un estudiante que no estaba 
vacunado de difteria. Dado que la respuesta no tenía por qué incluir su posicionamiento ante la obligatoriedad de la vacunación, se modificó la actividad para el 2016/17. En este caso la TD Social de la Fase 3 fue individual, acerca de la obligatoriedad de la vacuna de la difteria, tras una discusión grupal.

\section{Resultados}

\section{Modelo de sistema inmunológico construido}

Se presentan los resultados del 2015/16 en la Figura 2, y los del 2016/17 en la Figura 3. En las figuras se muestra la distribución del alumnado en los diferentes niveles del modelo, en las distintas fases de la secuencia (Fase 1: Inicio; Fase 2: Reestructuración; Fase 3: Transferencia). El tamaño de los círculos es proporcional al porcentaje de respuestas en cada nivel.

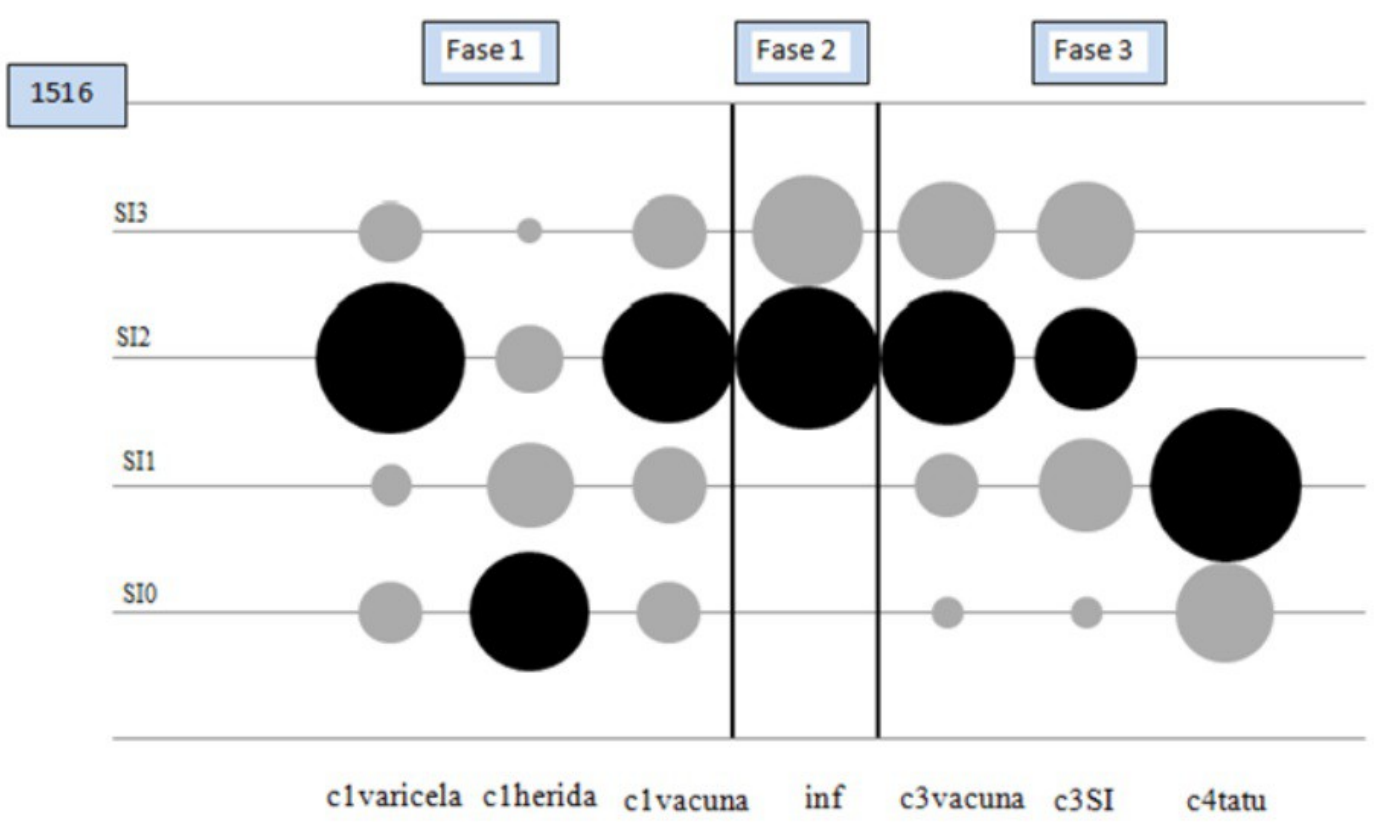

Figura 2. Niveles del modelo de sistema inmunológico expresados en las distintas actividades (2015/16).

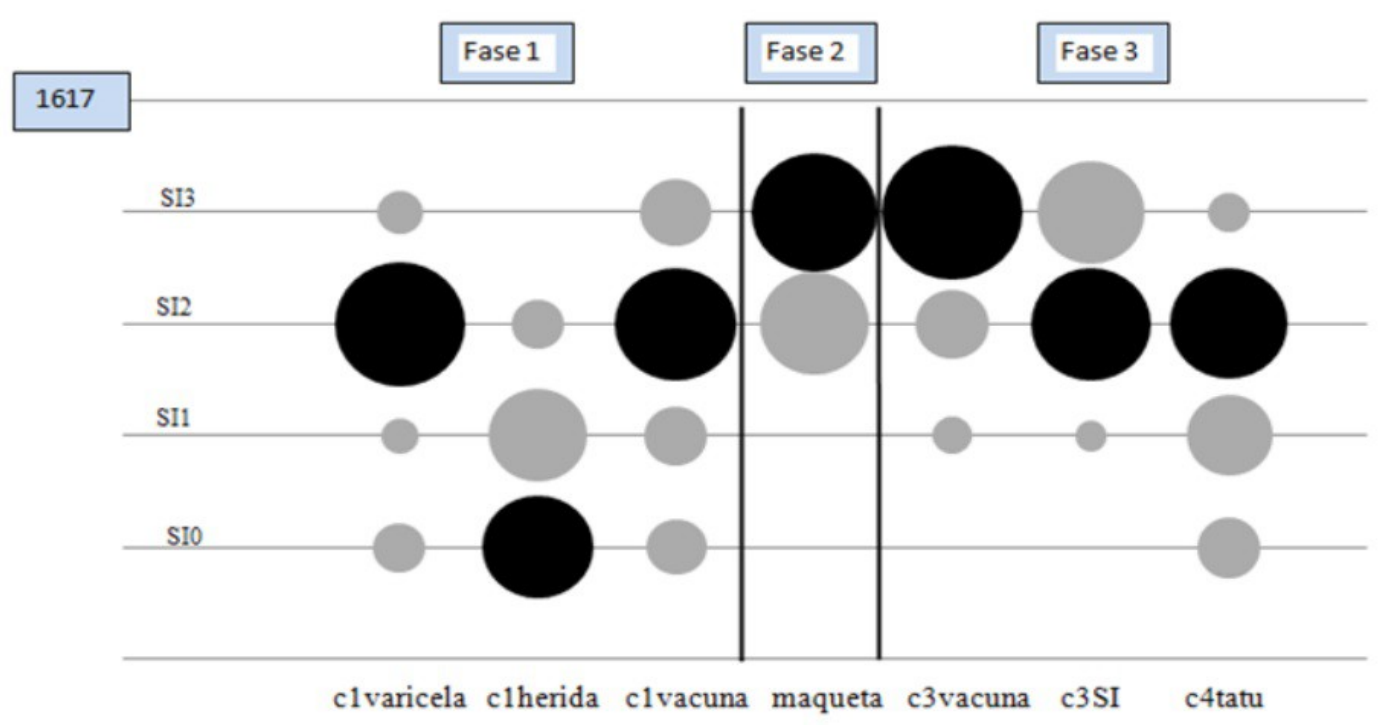

Figura 3. Niveles del modelo de sistema inmunológico expresados en las distintas actividades (2016/17). 
Se observa que en la Fase 1, en ambos cursos se dieron diferencias en los niveles del modelo dependiendo de los contextos, mostrándose un mayor nivel en las situaciones de la varicela y la vacuna, en las que se obtiene menor número de respuestas incorrectas. Sin embargo, en la pregunta referente a la herida, casi la mitad del alumnado no relacionó la infección de una herida con el funcionamiento del sistema inmunológico. Comparados los dos cursos, puede decirse que el nivel inicial de los grupos fue muy parecido.

Durante la Fase 2, el nivel del grupo de 2016/17 (57\% situado en el nivel SI3 y 43\% en el nivel SI2) fue superior al del curso anterior.

En cuanto a la transferencia del modelo a otros contextos, en la Fase 3, el alumnado de 2016/17 llevó a cabo una transferencia de mayor nivel tanto en el contexto cercano como en el lejano frente al grupo del curso 2015/16. En la transferencia cercana relacionada con la vacuna, se situó en los niveles SI2 y SI3, el 92\% en 2016/17 frente al 84\% en 2015/16; en la transferencia cercana relacionada con el funcionamiento del SI, el porcentaje de estudiantes en estos niveles fue el $96 \%$ en 2016/17 y 61\% en 2015/16. En el caso de la transferencia lejana en el contexto del tatuaje, se situaron en estos niveles el 44\% en 2016/17 frente al $0 \%$ en 2015/16.

En 2016/17 se observa que, en cuanto a número de estudiantes y porcentaje, hay un aumento en el nivel de complejidad de la construcción del modelo de sistema inmunológico de la Fase 1 a la Fase 3. Calculado el Tamaño de efecto (d de Cohen), los datos muestran que es significativo el tamaño de efecto en el caso del conocimiento sobre el funcionamiento de las vacunas $(\mathrm{d}=0,77)$, y del conocimiento sobre lo que ocurre en una herida $(\mathrm{d}=1,09)$. En 2015/16; sin embargo, estadísticamente se mantiene la hipótesis de que no hay efecto.

\section{Competencia argumentativa}

Se presentan los resultados obtenidos en cuanto a la competencia argumentativa en dos apartados. El primero corresponde a la calidad de la argumentación, teniendo en cuenta las justificaciones y limitaciones mostradas en los argumentos y el segundo, al tipo de justificaciones que manejan para posicionarse. Previamente a caracterizar la competencia argumentativa, se muestran los posicionamientos adoptados por los estudiantes, en la Tabla 3 respecto a la TD Personal en 2015/16 y 2016/17, y en la Tabla 4, el adoptado respecto a la TD Social.

Tabla 3. Distribución del posicionamiento de los estudiantes en la TD Personal en 2015/16 y 2016/17.

\begin{tabular}{|c|c|c|c|c|}
\hline & A favor & En contra & A favor & En contra \\
\hline \multirow{2}{*}{$2015 / 16$} & \multicolumn{2}{|c|}{ Fase $1(\mathrm{~N}=42)$} & \multicolumn{2}{|c|}{ Fase $3(\mathrm{~N}=37)$} \\
\hline & $97 \%$ & $2 \%$ & $67 \%$ & $30 \%$ \\
\hline \multirow{2}{*}{$2016 / 17$} & \multicolumn{2}{|c|}{ Fase $1(\mathrm{~N}=77)$} & \multicolumn{2}{|c|}{ Fase $3(\mathrm{~N}=75)$} \\
\hline & $92 \%$ & $4 \%$ & $83 \%$ & $13 \%$ \\
\hline
\end{tabular}


Tabla 4. Distribución del posicionamiento de los estudiantes en la TD Social en 2015/16 y 2016/17.

\begin{tabular}{|l|c|c|c|c|}
\cline { 2 - 5 } \multicolumn{1}{c|}{} & A favor & En contra & A favor & En contra \\
\hline \multirow{2}{*}{$2015 / 16$} & \multicolumn{2}{|c|}{ Fase 1 (N=42) } & \multicolumn{2}{c|}{ Fase 3 (N=9 grupos) } \\
\cline { 2 - 5 } & \multicolumn{2}{|c|}{$43 \%$} & $57 \%$ & \multicolumn{2}{c|}{ Fase 3 (N=75) } \\
\hline \multirow{2}{*}{$2016 / 17$} & \multicolumn{2}{|c|}{ Fase 1 (N=77) } & $72 \%$ & $28 \%$ \\
\cline { 2 - 5 } & $31 \%$ & $69 \%$ & & \\
\hline
\end{tabular}

\section{Calidad de la argumentación}

La Tabla 5 y la Tabla 6 recogen los datos relativos al promedio de justificaciones y limitaciones en los argumentos planteados en la TD Personal y en la TD Social, en 2015/16 y 2016/17 respectivamente.

Tabla 5. Promedios de justificaciones y limitaciones en los argumentos generados en las TD Personal y Social en 2015/16. (En cada Fase se muestran los resultados en conjunto y diferenciados según la postura adoptada: el gris claro indica $A$ favor; y el gris oscuro, En contra).

\begin{tabular}{|c|c|c|c|c|c|}
\hline & & \multicolumn{2}{|c|}{ Fase $1(\mathrm{~N}=42)$} & \multicolumn{2}{|c|}{ Fase $3(\mathrm{~N}=37)$} \\
\hline & & TODOS & $\begin{array}{c}\text { SEGÚN } \\
\text { POSTURA }\end{array}$ & TODOS & $\begin{array}{l}\text { SEGÚN } \\
\text { POSTURA }\end{array}$ \\
\hline \multirow{5}{*}{ TD Personal } & \multirow{2}{*}{ JUST } & \multirow{2}{*}{1,45} & 1,50 & \multirow{2}{*}{2,05} & 2,04 \\
\hline & & & 1 & & 2,27 \\
\hline & \multirow{3}{*}{ LIMIT } & \multirow{3}{*}{0,33} & 0,33 & \multirow{3}{*}{0,81} & 0,88 \\
\hline & & & & & \\
\hline & & & 1 & & 0,73 \\
\hline \multirow{5}{*}{ TD Social } & \multirow{3}{*}{ JUST } & \multirow{3}{*}{1,21} & 1,16 & \multirow{3}{*}{2,66} & \\
\hline & & & & & \\
\hline & & & 1,25 & & 2,66 \\
\hline & \multirow{2}{*}{ LIMIT } & \multirow{2}{*}{0,33} & 0,44 & \multirow{2}{*}{0,22} & \\
\hline & & & & & P? \\
\hline & & \multicolumn{2}{|c|}{ Fase $1(\mathrm{~N}=42)$} & \multicolumn{2}{|c|}{ Fase $3(\mathrm{~N}=9$ grupos) } \\
\hline
\end{tabular}


Tabla 6. Promedios de justificaciones y limitaciones en los argumentos generados en las TD Personal y Social en 2016/17. (En cada Fase se muestran los resultados en conjunto y diferenciados según la postura adoptada: el gris claro indica $A$ favor; el gris oscuro, En contra).

\begin{tabular}{|c|c|c|c|c|c|}
\hline & & Fase & $\mathrm{N}=74)$ & Fas & $\mathrm{N}=76)$ \\
\hline & & TODOS & $\begin{array}{c}\text { SEGÚN } \\
\text { POSTURA }\end{array}$ & TODOS & $\begin{array}{l}\text { SEGÚN } \\
\text { POSTURA }\end{array}$ \\
\hline \multirow{5}{*}{ TD Personal } & \multirow[t]{2}{*}{ JUST } & \multirow[t]{2}{*}{1,16} & 1,15 & \multirow[t]{2}{*}{1,78} & 1,82 \\
\hline & & & 1,33 & & 1,50 \\
\hline & \multirow{3}{*}{ LIMIT } & \multirow{3}{*}{0,36} & 0,37 & \multirow{3}{*}{0,97} & 0,98 \\
\hline & & & & & \\
\hline & & & 0 & & 0,9 \\
\hline \multirow{6}{*}{ TD Social } & \multirow{3}{*}{ JUST } & \multirow{3}{*}{1,05} & 1,13 & \multirow{3}{*}{2,10} & 2,04 \\
\hline & & & 1,02 & & 2,24 \\
\hline & & & & & \\
\hline & \multirow{3}{*}{ LIMIT } & \multirow{3}{*}{0,21} & 0,13 & \multirow{3}{*}{0,97} & 0,91 \\
\hline & & & & & \\
\hline & & & 0,25 & & 1,14 \\
\hline & & \multicolumn{2}{|c|}{ Fase $1(\mathrm{~N}=77)$} & \multicolumn{2}{|c|}{ Fase $3(\mathrm{~N}=75)$} \\
\hline
\end{tabular}

Respecto a la TD Personal, en 2015/16, tanto las personas que deciden poner la vacuna contra el virus del papiloma humano en la Fase 3 como las que deciden no ponerla utilizan un mayor número de justificaciones a la hora de defender su posicionamiento que en la Fase 1. De la misma manera, aumenta la consideración de las limitaciones de la decisión tomada. El tamaño de efecto ( $\mathrm{d}$ de Cohen) estadístico es moderado para ambos indicadores $(\mathrm{d}=0,49$ para las justificaciones y d=0,44 para las limitaciones). En 2016/17 la situación de partida es parecida en cuanto a limitaciones y algo inferior en cuanto a justificaciones. Sin embargo, se observan las mismas mejoras en la Fase 3, y el tamaño de efecto de la secuencia es mayor: $d=0,75$ para las justificaciones y $\mathrm{d}=0,59$ para las limitaciones.

En cuanto a la TD Social, en 2015/16 hay que tener en cuenta que en la Fase 3 los estudiantes actuaron en grupo, por lo que no se puede interpretar de la misma manera el aumento en el número de justificaciones. Sí que puede resultar llamativo por este mismo motivo el bajo promedio de limitaciones expresadas. En 2016/17, en la Fase 3 se observa un aumento considerable de las justificaciones y limitaciones expresadas. De hecho, el tamaño de efecto (d de Cohen) estadístico muestra que el efecto es grande en ambos casos: $\mathrm{d}=1,02$ para las justificaciones y $\mathrm{d}=0,86$ para las limitaciones. 


\section{Tipos de justificaciones}

En la figura 4 se muestran todas las categorías de justificaciones utilizadas por los estudiantes que se posicionan en un sentido u otro en la TD Personal y en la TD Social, en 2015/16. Se han tenido en cuenta tanto las utilizadas para justificaciones como para limitaciones. En la Figura 5 se hace lo propio en 2016/17.

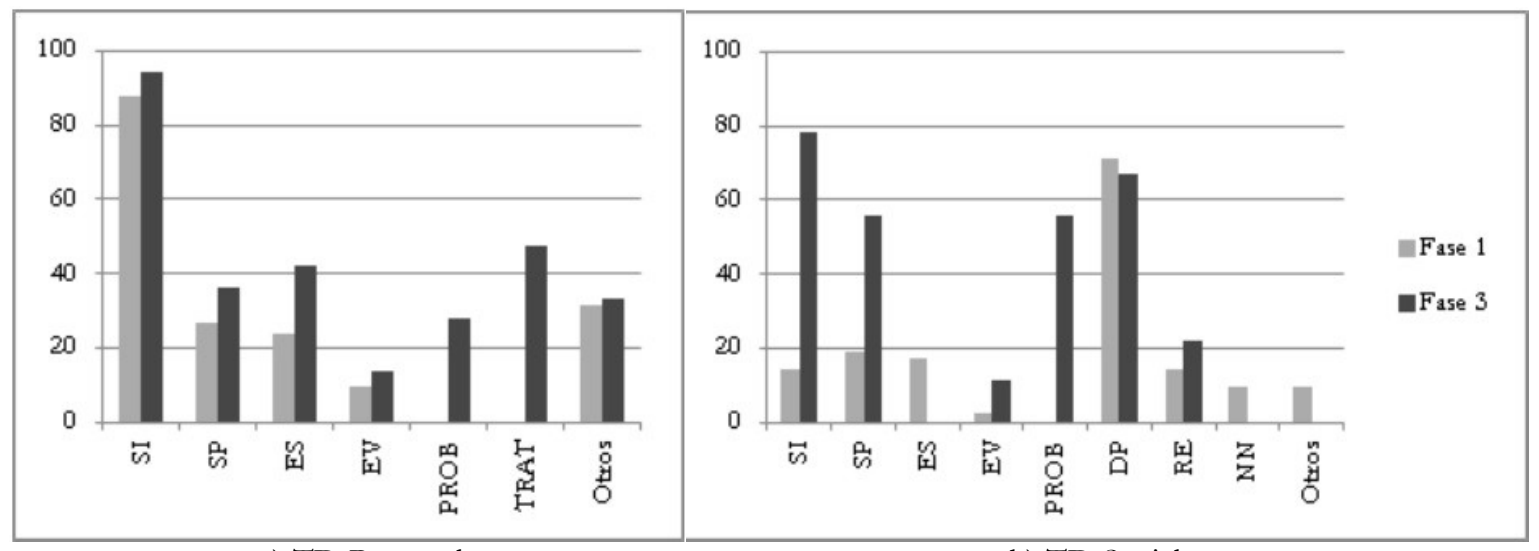

a) TD Personal

b) TD Social

Figura 4. Categorías de justificaciones y limitaciones empleadas por los estudiantes durante la Fase 1 y la Fase 3 en 2015/16 en su posicionamiento. Se indica el porcentaje de estudiantes que alude a cada categoría (SI: Salud individual; SP: Salud pública; ES: Efectos secundarios; EV: Eficacia de las vacunas; PROB: Probabilidad de desarrollar la enfermedad; TRAT: Tratamientos relacionados con el papiloma; DP: Decisión Personal; RE: Recomendación de expertos; NN: No son naturales).

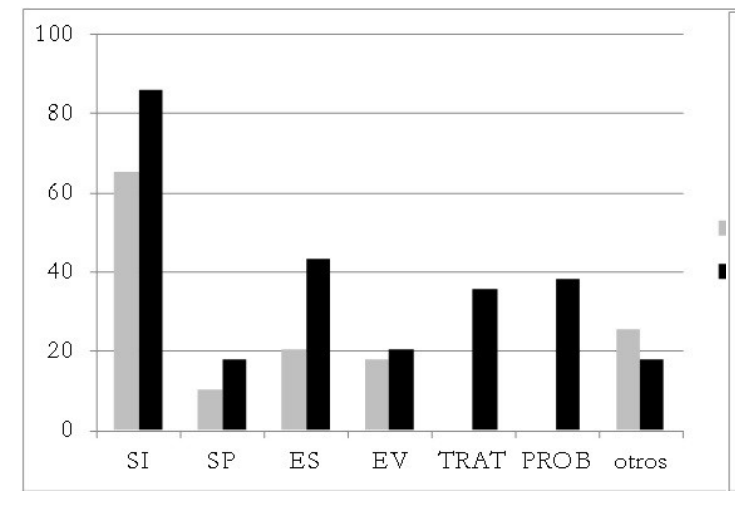

a) TD Personal

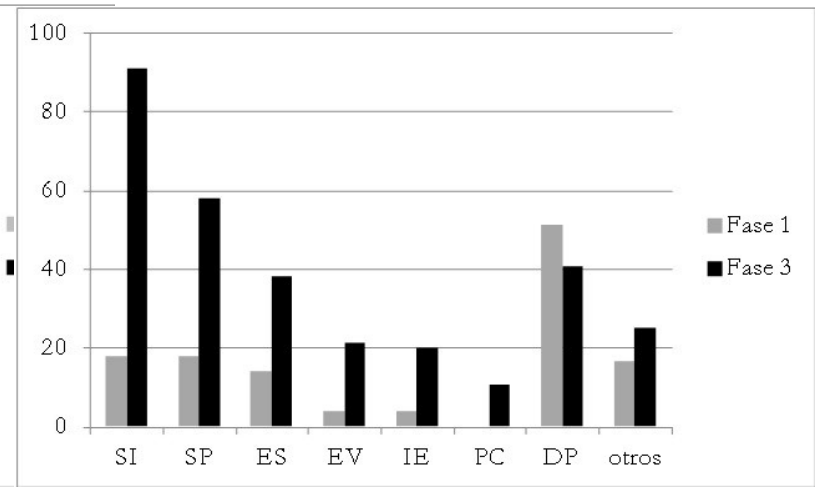

b) TD Social

Figura 5. Categorías de justificaciones y limitaciones empleadas por los estudiantes durante la Fase 1 y la Fase 3 en 2016/17 en su posicionamiento. Se indica el porcentaje de estudiantes que alude a determinada categoría (SI: Salud individual; SP: Salud pública; ES: Efectos secundarios; EV: Eficacia de las vacunas; TRAT: Tratamientos relacionados con el papiloma; PROB: Probabilidad de desarrollar la enfermedad; IE: Intereses económicos; PC: Pruebas científicas; DP: Decisión personal)

En cuanto a la TD Personal, tanto en 2015/16 como 2016/17, la justificación más utilizada desde un inicio es la Salud individual. Teniendo en cuenta el posicionamiento adoptado, la principal justificación para defender la vacunación ha sido la Salud individual, seguida de la Salud pública aún teniendo en cuenta los Efectos secundarios. El alumnado contrario a la vacunación, menciona los Efectos secundarios y la existencia de Tratamiento diferente a la vacunación frente a la enfermedad del papiloma humano (50\% en la Fase 3 de 2016/17). 
En la TD Social, la situación cambia de la Fase 1 a la Fase 3. Así, la Decisión personal destaca en la Fase 1, mientras que en la Fase 3 aumentan de forma importante la alusión a la Salud individual y a la Salud pública. De hecho, en 2016/17 el tamaño de efecto es significativo para Salud individual $(\mathrm{d}=1,32)$, Salud pública $(\mathrm{d}=0,74)$ y Efectos secundarios $(\mathrm{d}=0,43)$. En la Fase 3, el alumnado a favor de la obligatoriedad maneja como justificación la Salud individual, mientras que el alumnado que está en contra alude a las libertades individuales, a respetar la Decisión personal.

\section{Discusión de resultados y conclusiones}

Los resultados obtenidos señalan que el modelo de sistema inmunológico construido por los estudiantes ha alcanzado un mayor nivel de complejidad durante el curso 2016/17, a pesar de que el nivel inicial es similar al del curso 2015/16. Las profesoras durante este curso han actuado en mayor medida como guías de la actividad cognitiva del alumnado, en su andamiaje, en sus dos elementos clave (Hardy et al. 2006): han estructurado las actividades para mantener a los estudiantes centrados en los aspectos relevantes del modelo y han facilitado la reflexión del alumnado sobre aspectos comunes de los modelos representativos de las situaciones contextualizadas. El andamiaje favorecido por las profesoras en torno a estos dos elementos ha propiciado unos mejores resultados en el aprendizaje. Esta conclusión es similar a la formulada en otros estudios (Decristan et al. 2015, Hardy et al. 2006, Zangori et al. 2015), de manera que se puede considerar que la guía en el proceso de aprendizaje puede ser imprescindible (Kirschner et al. 2006). En cuanto a las actividades, parece que el haber pensado sobre distintas situaciones contextualizadas en las que actúa el sistema inmunológico (vacuna, enfermedades infecciones, alergias, herida) y la realización de una representación tridimensional de los procesos del cuerpo de cada una de ellas ha ayudado, en mayor medida que el simple intercambio de información, a la construcción de un modelo más adecuado. Unos mejores resultados en la transferencia cercana y lejana del modelo de sistema inmunológico en 2016/17 indican, al igual que otros estudios (Izquierdo, Caamaño y Quintanilla 2007; Marchán-Carvajal y Sanmartí 2014) que la utilización de diversos contextos donde se presenta un mismo modelo favorece la interrelación entre distintos fenómenos científicos y favorece la transferencia (Bransford y Schwartz 1999).

En cuanto a la facilitación de la reflexión del alumnado por parte de las profesoras, la labor de las profesoras (sobre todo durante los momentos de discusión en cada grupo sobre el modelo representativo de la situación contextualizada y en el momento de la generalización del modelo consensuado sobre el sistema inmunológico del aula), puede haber propiciado que los estudiantes mantuvieran el modelo más estable en el tiempo. La estabilidad del modelo mental de los estudiantes justificaría su aplicación a nuevas situaciones incluso cuando se ha solicitado una transferencia lejana (el 44\% del alumnado se ha situado en un nivel mínimo de SI2 en la transferencia lejana). Este estudio avala pues los resultados de otras investigaciones donde se destaca el papel desempeñado por la persona docente en la construcción del modelo (Justi 2006) y la estabilidad en la transferencia (Burgoa 2014) o en la abstracción y generalización (Schalk et al. 2013). Una limitación del presente estudio es que no se dispone de datos que permita discernir en qué medida ha favorecido cada uno de los elementos en la guía prestada por las profesoras (actividades y facilitación) la construcción y transferencia del modelo por parte de los estudiantes.

En cuanto a la competencia argumentativa durante la toma de decisiones sobre la vacunación, los estudiantes tienen en cuenta más aspectos relacionados tanto con las ventajas como con las desventajas de las vacunas para posicionarse, es decir, utilizan más justificaciones para respaldar la opción que escogen, así como limitaciones de la opción escogida lo que indica una 
alta calidad de la argumentación (Erduran et al. 2004; García-Milá et al. 2013). El tamaño de efecto de la secuencia es grande en la TD Social en 2016/17, moderado en la TD Personal en 2016/17 y entre bajo y moderado en la TD Personal en 2015/16. Sería interesante poder hacer la comparación entre los dos cursos para la TD Social, pero en 2015/16 en la Fase 3 los estudiantes actuaron en grupo, por lo que los resultados no son comparables.

En cuanto al tipo de justificaciones y limitaciones que el alumnado ha tenido en cuenta, si bien, tanto en la TD Personal como en la TD Social, los porcentajes suben en la Fase 3, es en la TD Social de 2016/17 donde el aumento del uso de determinadas categorías (Salud individual, Salud pública, Efectos secundarios) es significativo. Se puede pensar que la construcción de conocimiento sobre el funcionamiento del sistema inmunológico a lo largo de la secuencia puede haber influido en el aumento de la consideración de estas categorías. De esta manera, parece que el trabajo realizado por las profesoras en 2016/17 ha facilitado que el alumnado construya un modelo más completo, pero también que lo aplique a la hora de argumentar sus tomas de decisión. De todas formas, este aspecto necesita ser analizado, ya que ni siquiera se puede realizar el contraste con lo ocurrido en 2015/16.

Por otra parte, los datos de 2016/17 muestran que ha habido diferencias entre las dos tomas de decisión, y que en la de ámbito social, los estudiantes han utilizado más justificaciones para defender su opción. Esto puede deberse a que son conscientes de que las implicaciones de esta toma de decisión afectan a toda la sociedad. Así, en la TD Social, el tipo de justificaciones utilizado ha sido diferente según la opción escogida. Destaca la Salud individual como justificación de los estudiantes que defienden las vacunas y su obligatoriedad, mientras que el tipo de justificación de las personas que no apoyan la obligatoriedad de las vacunas es la alusión a la libertad individual (Decisión Personal), un punto sobre el que discuten en varios grupos y que merece ser discutido explícitamente en cuestiones controvertidas de este tipo, el alcance de las libertades individuales en una convivencia compartida.

Los aspectos que se concluyen de este estudio merecen ser analizados con cuidado en futuras investigaciones. Se ha visto que el profesorado, a través de la propuesta de actividades y de las intervenciones, ha favorecido la construcción y transferencia del modelo. Sin embargo, como se ha señalado, sería interesante analizar en qué medida lo ha hecho cada uno de los elementos. Se podría, por ejemplo, caracterizar las intervenciones del profesorado y analizar la influencia de los distintos tipos de intervenciones en la construcción del modelo (Justi 2006). Por otra parte, este estudio se ha centrado en ver la influencia del profesorado en la construcción de conocimiento pero no se ha analizado cómo puede ayudar el profesorado a que los estudiantes desarrollen su competencia argumentativa. En este caso, los datos parecen indicar que la mejora en el conocimiento conceptual acerca del sistema inmunológico se ha traducido en que los estudiantes utilizan los conocimientos conceptuales en las tomas de decisión, bien como justificaciones, bien como limitaciones, desempeñando una mejor argumentación. Esta posible relación entre la argumentación y el conocimiento conceptual ha sido analizada en otras investigaciones, hallando que es necesario un conocimiento conceptual mínimo para poder elaborar argumentos (Sadler y Donnelly 2006) y para que el argumentar pueda facilitar la profundización en el conocimiento (von Aufschnaiter, Erduran, Osborne y Simon 2008). Las autoras están analizando cómo es la relación entre los dos desempeños en este caso, de qué manera el conocimiento conceptual es aplicado en las tomas de decisión, si se trata de dos competencias totalmente independientes o si hay un posible umbral de mínimo conocimiento para argumentar. 


\section{Agradecimientos}

Al proyecto de investigación EHU15/25, financiado por la UPV/EHU, del que forma parte este trabajo. Al proyecto EDU2015-66643-C2-1-P, fianciado por el Ministerio de Economía y Competitividad. Al alumnado que participó en la investigación.

\section{Referencias}

Alexander P.A., Murphy P.K. (1999) Nurturing the seeds of transfer: A domain-specific perspective. International Journal of Educational Research 31 (7), 561-576.

Alzate Ó.E.T. (2013) Modelos y modelización en la enseñanza y el aprendizaje de las ciencias. Enseñanza de las Ciencias Número extra, 3484-3487.

Andrade V.A., Araújo-Jorge T.C., Coutinho-Silva R. (2016) Concepções discentes sobre inmunologia e sistema immune humano. Investigações em Ensino de Ciências 21 (3), 1-22. https://www.if.ufrgs.br/cref/ojs/index.php/ienci/article/view/144/235

Aznar V., Puig B. (2016a) Concepciones y modelos del profesorado de primaria en formación acerca de la tuberculosis. Enseñanza de las Ciencias 34(1), 33-52. https://doi.org/10.5565/rev/ensciencias. 1670

Aznar,V., Puig B. (2016b) ¿Qué conocimientos movilizan un grupo de futuros docentes para ela- borar el modelo de infección por tuberculosis? Revista Eureka sobre Enseñanza y Divulgación de las Ciencias 13(2), 264-278.

Barnett S.M., Ceci S.J. (2002) When and where do we apply what we learn? A taxonomy for far transfer. Psychological Bulletin 128 (4), 612-637.

Bransford J.D., Schwartz D.L. (1999) Rethinking transfer: A simple proposal with multiple implications. Review of Research in Education 24 (3), 61-100.

Burgoa B. (2014) La transferencia de contenidos matemáticos a contextos cientificos: el concepto de función. Tesis doctoral. Universidad del País Vasco/Euskal Herriko Unibertsitatea. Bilbao, España.

Carpintero E. (2002) El proceso de transfer: Revisión y nuevas perspectivas. EduPsykhé: Revista de Psicología y Psicopedagoía 1 (1), 69-96.

Criswell B. (2012) Framing inquiry in high school chemistry: Helping students see the bigger picture Journal of Chemical Education 89, 199-205.

Decristan J., Hondrich A.L., Büttner G., Hertel S., Klieme E., Kunter M., Lühken A., AdlAmini K., Djakovic S.-K., Mannel S., Naumann A., Hardy I. (2015) Impact of additional guidance in science education on primary students' conceptual understanding. The Journal of Educational Research 108 (5), 358-370.

Erduran S., Simon S., Osborne J. (2004) TAPping into argumentation: Developments in the application of Toulmin's Argument Pattern for studying science discourse. Science Education 88 (6), 915-933.

Erickson F. (1989) Métodos cualitativos de investigación sobre la enseñanza. En M. Wittrock (Ed.), La investigación de la enseñanza, II. Métodos cualitativos y de observación (pp. 195-301). Barcelona: Paidós.

García-Milá M., Gilabert S., Erduran S., Felton M. (2013) The effect of argumentative task goal on the quality of argumentative discourse. Science Education 97 (4), 497-523.

Gee J.P. (1999) An introduction to discourse analysis: Theory and method. Londres: Routledge. 
Gilbert J.K. (2004) Models and Modelling: Routes to More Authentic Science Education. International Journal of Science and Mathematics Education 2 (2), 115-130.

Hardy I., Jonen A., Möller K., Stern E. (2006) Effects of instructional support within constructivist learning environments for elementary school students' understanding of "floating and sinking". Journal of Educational Psychology 98, 307-326. doi:10.1037/00220663.98.2.307.

Izquierdo M., Caamaño A., Quintanilla M. (2007) Investigar en la enseñanza de la química. Nuevos horizontes: Contextualizar y modelizar. http://edumat. uab. cat/didactica/files/compartits/28. pdf

Jiménez-Aleixandre M.P. (2010) 10 ideas clave. Competencias en argumentación y uso de pruebas. Barcelona: Graó.

Justi R. (2006) La enseñanza de ciencias basada en la elaboración de modelos. Enseñanza de las Ciencias 24 (2), 173-184.

Kirschner P.A., Sweller J., Clark R.E. (2006) Why minimal guidance during instruction does not work: An analysis of the failure of constructivist, discovery, problem-based, experiential, and inquiry-based teaching. Educational Psychologist 41(2), 75-86.

Kortland K. (1996) An STS case study about students'decision making on the waste issue. Science Education 80 (6), 673-689.

Lobato J. (2012) The actor-oriented transfer perspective and its contributions to educational research and practice. Educational Psychologist 47(3), 232-247.

Maguregi G., Uskola A., Burgoa B. (2017) Modelización, argumentación y transferencia de conocimiento sobre el sistema inmunológico a partir de una controversia sobre vacunación en futuros docentes. Enseñanza de las Ciencias 35 (2), 29-50. http://dx.doi.org/10.5565/rev/ensciencias.2237

Marchán-Carvajal I., Sanmartí N. (2015) Criterios para el diseño de unidades didácticas contextualizadas: aplicación al aprendizaje de un modelo teórico para la estructura atómica. Educación Química 26, 267-274.

Marchán-Carvajal I., Sanmartí N. (2014) Una revisión sobre el uso de contextos en la enseñanza de las ciencias y su potencial para el desarrollo de la competencia científica. En De las Heras et. al. (coord.). Investigación y transferencia para una educación en ciencias: Un reto emocionante (pp. 702-710). Huelva: Servicio de Publicaciones de la UHU.

Mendonça P.C.C., Justi R. (2014) An instrument for analyzing arguments produced in modeling-based chemistry lessons. Journal of Research in Science Teaching 51 (2), 192-218.

Morales P. (2011) El tamaño del efecto (effect size): análisis complementarios al contraste de medias. $\%$ F1oDelEfecto.pdf

NRC (2012) A Framework for K-12 Science Education: Practices, Crosscutting conceps, and Core ideas. https://www.nap.edu/read/13165/chapter/1

OCDE (2013) PISA 2015. Draft science framework. https://www.oecd.org/pisa/pisaproducts/Draft \%20PISA\%202015\%20Science $\% 20$ Framework $\% 20$.pdf

Pigrau T., Sanmartí N. (2015) Model per interpretar sistemes vius. Recuperado de: http://media.wix.com/ugd/81d0d8_2bd060dd60e84ba88ed018a28dc03fe6.pdf 
Sadler T.D., Donnelly L.A. (2006) Socioscientific argumentation: The effects of content knowledge and morality. International Journal of Science Education 28 (12), 1463-1488.

Schalk H.H., van der Schee J.A., Boersma K.Th. (2013) The development of understanding of evidence in pre-university biology education in the Netherlands. Research in Science Education 43, 551-578.

Schwarz C.V., Reiser B.J., Davis E.A., Kenyon L., Achér A., Fortus D., Shwartz Y., Hug B., Krajcik J. (2009) Developing a learning progression for scientific modeling: Making scientific modeling accessible and meaningful for learners. Journal of Research in Science Teaching, 46 (6), 632-654.

Toulmin S. (1958) The uses of argument. Cambridge, England: Cambridge University Press.

von Aufschnaiter C., Erduran S., Osborne J., Simon S. (2008) Arguing to learn and learning to argue: Case studies of how students' argumentation relates to their scientific knowledge. Journal of Research in Science Teaching 45 (1), 101-131.

Zangori L., Forbes C.T., Schwarz C.V. (2015) Exploring the effect of embedded scaffolding within curricular tasks on third-grade students' model-based explanations about hydrologic cycling. Science \& Education 24, 957-981. 